\title{
Tooth Aspiration in Child during a Dental Procedure: Case Report and Review of the Literature
}

\author{
Amina Hadžibeganović ${ }^{1}$, Husref Tahirovićn ${ }^{2}$ Nasih Halilbašić $^{3 *}$ \\ ${ }^{1}$ Clinic for Oncology and Radiotherapy, University Clinical Centre Tuzla, Tuzla, Bosnia and Herzegovina; ${ }^{2}$ Department \\ of Medical Sciences of the Academy of Sciences and Arts of Bosnia and Herzegovina, Sarajevo, Bosnia and Herzegovina, \\ ${ }^{3}$ Department of Pediatrics, University Clinical Center Tuzla, Tuzla, Bosnia and Herzegovina,
}

Correspondence: husref.tahirovic@gmail.com; Tel./Fax.: + 38735303740

Received: December 9, 2019; Accepted: February 12, 2020

\begin{abstract}
Objective - The aim of this article is to illustrate the importance of a prompt evaluation of a foreign body aspiration (FBA). Case Presentation - A 13-year old boy was admitted to hospital in 1981, one and a half month after a dental procedure of tooth extraction. The tooth was presumed to have been ingested during the procedure and the child was sent home. The symptoms presented immediately and were aggravating during the time. Before the admission to the hospital the child was examined by medical professionals on three occasions but only at hospital the FBA was suspected. During the first bronchoscopy, the foreign body had not been extracted, so the child was transferred to the referral centre where the tooth was successfully removed and he recovered. Conclusion - In the setting of dental procedure in paediatric population, the missing instrument, material or extracted tooth must be found or a foreign body aspiration and ingestion should be suspected until proven otherwise.
\end{abstract}

Key Words: Foreign Body Aspiration - Tooth • Dental Procedure.

\section{Introduction}

Foreign body aspiration (FBA) represents an accidental threat to breathing due to inhalation or ingestion of food or other objects causing obstruction of respiratory tract (1). FBA can be seen in all age groups, but is the most prevalent (up to $75 \%$ ) in children between the ages 0 and 3, with male to female ratio 3:2 (2). Organic foreign bodies are more common than inorganic. The exact type of foreign body has to do with the cultural context, but in general, among the food items nuts and seeds are involved most frequently, while among the nonfood items of highest prevalence are round-shaped

"Nasih Halilbašić (1938-2003) was one of the most prominent pediatric pulmonologists in Bosnia and Herzegovina in the late twentieth century. objects (3). The important aspect is that FBA and its complications are preventable.

In order to illustrate the importance of a prompt evaluation of FBA, we present this case of tooth aspiration during a dental procedure.

\section{Case presentation}

We report this case of a 13-year-old boy with FBA, from the year 1981. The symptoms occurred one and a half month before the admission to hospital, after the dental procedure of tooth extraction. According to the report, the child ingested the tooth during the procedure, started coughing, but was sent home. The cough was persistent and 5 to 6 days later he had a fever. That was the first time the child was examined by a medical profes- 


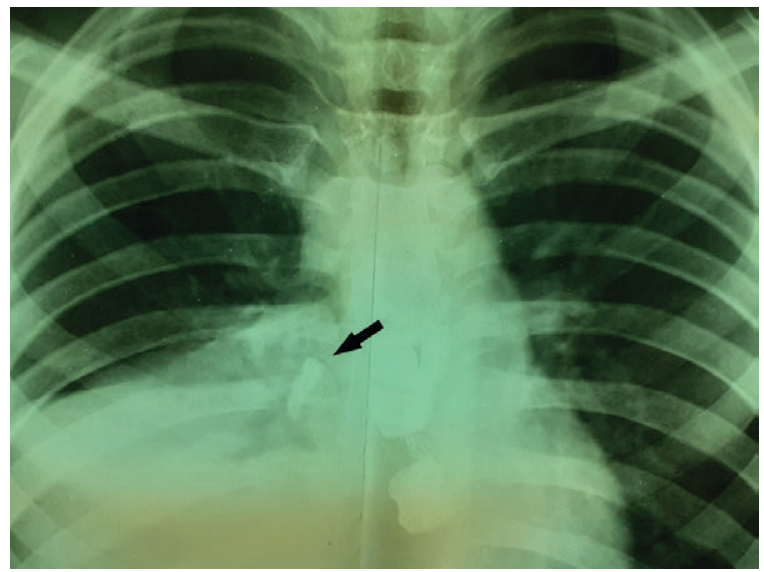

Fig. 1. Chest X-ray Performed at the Admission Shows Infiltration, Pleural Effusion and the Shadow in Shape of a Tooth on the Right Side.

sional, when, according to the information given by mother during the admission to the hospital, he was prescribed a 5-day course of some capsules and syrup against cough. During the follow-up examination he seemed better, but the symptoms did not stop. He was coughing persistently and had a fever. He was also exhausted, refusing food and started losing weight. Mother was giving him antipyretics. The day before he was admitted to the hospital, he had been examined, had had a chest X-ray and had been sent to hospital as an exudative interlobular pleuritis to the right.

At the admission to the hospital, the child was conscious, had fever $\left(38^{\circ} \mathrm{C}\right)$, was pale, intoxicated, malnourished, with high respiratory rate (64 per minute), dyspnoeic (widening of nostrils during inspiration, retraction of intercostal spaces and jugular pit), without cyanosis. The tongue was moist with white deposit in the middle line. Pulsations on the neck bilaterally and in the second intercostal space to the left were visible. The right hemithorax was slightly falling back during respiration and breath sounds were decreasing from the top to the bottom, where they were silent. Heart rate was 124 per minute. There were no other significant findings during the examination.

Significant lab findings at the admission were: ESR $133 / 137 \mathrm{~mm} / \mathrm{h}$; WBC $20,0 \times 10^{9}$ cells $/ \mathrm{L}$; Hb

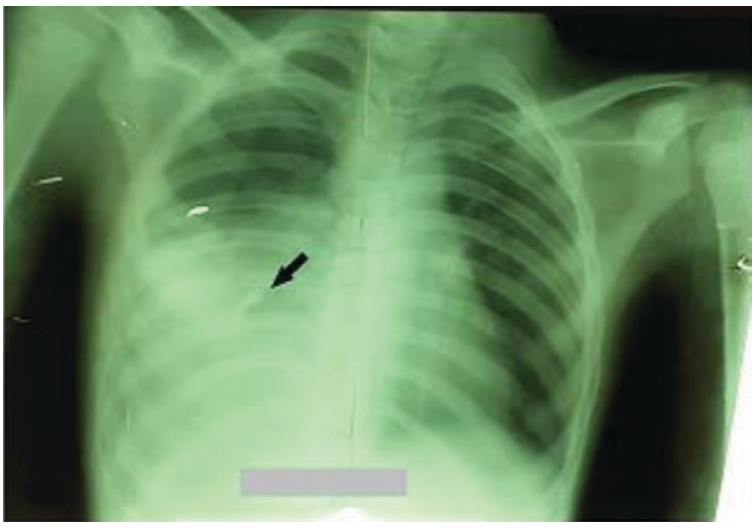

Fig. 2. Chest X-ray Performed after the Bronchoscopy Show That the Tooth Was Not Extracted but Dislocated.

$56 \mathrm{~g} / \mathrm{L}$; some proteins and 4-6 leucocytes in urine. Blood culture taken at the admission came positive for Staphylococcus albus saprophyticus. The chest Xray was also taken at the admission (Fig. 1)

At the admission penicillin was administered intramuscularly and the intravenous rehydration was started. Antipyretics and oxygen were administered occasionally as needed. On the second day, the fever (up to $39.8^{\circ} \mathrm{C}$ ) continued, so the intravenous course of methicillin and gentamycin was started. The fever dropped after the antibiotics had been administered. The child received transfusion of deplasmated red blood cells due to severe anaemia. Bronchoscopy under general anaesthesia was performed in order to extract the foreign body. During bronchoscopy, pus was found in trachea. In the right principal bronchus apart from the pus, there were granulations. The granulations were partially removed so the foreign body (most likely the tooth), lodged in the oedematous wall of the bronchus, was seen. Despite several tries, the extraction of the foreign body was impossible. After the bronchoscopy the chest X-ray was retaken (Fig. 2). The thoracic surgeon proposed an urgent transfer to the referral centre. On the third day, the child was afebrile and the intravenous antibiotic course was continued. The child was transferred to the medical institution specialised in lung disease where the toot was removed via bronchoscopy. 


\section{Discussion}

The factors that predispose preschool children to FBA are inadequate observation by adults, tendency of children to explore environment through their mouth, the fact that children often run and play during the food ingestion, inability to chew food, inadequately developed posterior dentition and immature neuromuscular mechanisms of airway protection $(4,5)$. The FBA is a potentially lethal event that is more prevalent than reported. According to the Computer-Assisted Telephone Interview survey in Italy, around $80 \%$ of children below the age of 15 who incur foreign body injury are treated in emergency department or hospital and the others do not seek for medical help (6). The most prevalent foreign body aspirates in Bosnia and Herzegovina are peanuts and pumpkin seeds, but differently from the statistics in the other European countries, female children are more prone to the foreign body injuries than male (3). A tooth in the airway is an extremely rare foreign body, especially in the setting of aspiration during a dental procedure $(4,7)$. A 10-year institutional review at The University of North Carolina reported 36 cases of aspiration and ingestion in dental practice of which only one was a proven case of aspiration (8). The clinical presentation of FBA depends primarily on the characteristics of foreign body and its anatomic location, but also the time elapsed between the FBA and obtained medical care. Upper airway involvement varies from complete obstruction with hypoxia and cardiorespiratory compromise to partial obstruction with stridor, coughing, wheezing, and respiratory distress, while foreign bodies located in the lower airway lead to pulmonary changes dependent on the type of impaction (2). Since the symptoms and signs of FBA vary, a clinical algorithm for management of suspected FBA in children was proposed in 2017. According to this algorithm there are five findings that are scored: (a) Witnessed choking; (b) Noisy breathing/stridor/ dysphonia; (c) New onset/recurrent/persistent wheeze; (d) Unilateral reduced air entry; and (e) Abnormal chest X-ray.
Total score directs the further management (9). The value of chest X-ray findings depends on the radiopacity of the foreign body and since the organic foreign bodies are the most prevalent, the radiological findings are often normal in cases of FBA. Possible radiological findings are hyperlucency/obstructive emphysema, mediastinal shift, localised atelectasis and pulmonary infiltration (10). Some authors point out the possible value of reduced or absent lung sliding on a lung ultrasound in evaluation of suspected FBA (11). The most common complications of FBA are air-trapping, pneumonia, atelectasis, but pneumomediastinum, pneumothorax, bronchiectasis and lung abscess can also be seen. The occurrence rate of air-trapping, pneumonia and atelectasis is significantly higher in cases of delayed treatment (12). A study surveyed in People's Republic of China showed that most of the cases of FBA (63.3\%) seek medical care within 1 to 3 days. Only $18.4 \%$ visit doctor within 24 hours and significant $4.6 \%$ ask for help one month or more after the FBA. According to this study the delay in visit time is in correlation with the educational level of caretaker and the further evaluation once the child gets into the healthcare system depends significantly on the fact whether the caretakers state the possibility of FBA or not (13). Method of choice for removal of foreign body from airway is rigid bronchoscopy under general anaesthesia (14).

This report presents a rare case of FBA during a dental procedure. The tooth was presumed to be ingested despite the fact that the child started coughing right after. Details from personal and family history suggest that this child lived in poor social conditions, which could be one of the causes of a 1.5-month delay in treatment in this case. The other cause of this delay is the fact that three visits to the healthcare institution that had antedated the admission to hospital had lacked the information about the possibility of FBA, so the child had been treated for the respiratory infection. This matches the literature findings that pneumonia, bronchitis and upper respiratory tract infections are most common primary diagnosis among the misdiag- 
nosed patients with FBA (12). When it comes to the tooth as an aspirated foreign body, the problem could be in fact that the medical professionals are usually well aware of the risk of aspiration of natal and neonatal teeth, but generally assume that children above 6 years are not at high risk of aspiration of primary teeth, so tooth aspiration is rarely taken into consideration as a possible diagnosis (15). All the complications in this case could have been avoided if it had not been presumed that the child ingested the tooth during the dental procedure.

\section{Conclusion}

In the setting of dental procedure in paediatric population, the missing instrument, material or extracted tooth must be found or a foreign body aspiration and ingestion should be suspected until proven otherwise.

\section{Learning Points}

- Tooth aspiration is rare but possible in all age groups and under different circumstances including dental procedures.

- The FBA is often misdiagnosed for respiratory infections. If the symptoms continue after the treatment, the FBA should be suspected.

- Delay in treatment of FBA depends on different factors and can be fatal.

- If the FBA during dental procedure is not prevented, further complications are preventable by prompt evaluation.

Acknowledgement: Late primarius Dr. Nasih Halilbašić, who recorded this history.

Conflict of Interest: The authors declare that they are members of the Editorial Team of the Central European Journal of Paediatrics.

\section{References}

1. ICD-10 Version 2016 [homepage on the Internet] [cited 2019 Nov 25]. Available from: https://icd.who.int/ browse10/2016/en\#/W79.
2. Foltran F, Ballali S, Rodriguez H, Van As AB, Passali D, Gulati A, et al. Inhaled Foreign Bodies in Children: A Global Perspective on Their Epidemiological, Clinical, and Preventive Aspects. Pediatr Pulmonol. 2013;48(4):34451. doi: 10.1002/ppul.22701.

3. Lorenzoni G, Umihanić Š, Azzolina D, Manza E, Brkić F, Gregori D. A novel approach for comparing patterns of foreign body injuries across countries: A case study comparing European Countries and Bosnia and Herzegovina. Int J of Pediatr Otorhinolaryngol. 2018;105:90-6. doi: 10.1016/j.ijporl.2017.12.005.

4. Swain SK, Sahoo S, Mahesh CS. From tooth extraction to fatal airway complication in a child - A Case report. EJENTAS. 2016; 17(1):27-9.

5. Foreign Body Aspiration in Infants and Older Children: A comparative Study. Na'ara, S, Vainer I, Amit M, Gordin A. Ear Nose Throat J [serial on the Internet]. 2019 Apr [cited 2019 Dec 25]; $145561319839900:[5$ p.]. Avalaible from: https://journals.sagepub.com/doi/ pdf/10.1177/0145561319839900.

6. Snidero S, Soriani N, Baldi I, Zobec F, Berchialla P, Gregori D. Scale-up Approach in CATI Surveys for Estimating the Number of Foreign Body Injuries in the Aero-digestive Tract in Children. Int J Environ Res Public Health. 2012;9(11):4056-67. doi: 10.3390/ijerph9114056.

7. Hou R, Zhou H, Hu K, Ding Y, Yang X, Xu G et al. Thorough documentation of the accidental aspiration and ingestion of foreign objects during dental procedure is necessary: review and analysis of 617 cases. Head Face Med. 2016;12(3):23. doi: 10.1186/s13005-016-0120-2.

8. Tiwana KK, Morton T, Tiwana PS. Aspiration and ingesstion in dental practice: A 10-year institutional review. J Am Dent Assoc. 2004;135(9):1287-91.

9. Janahi IA, Khan S, Chandra P, Al-Marri N, Saadoon A, Al-Naimi L, et al. A new clinical algorithm scoring for management of suspected foreign body aspiration in children. BMC Pulm Med. 2017;17(1):61-71. doi: 10.1186/ s12890-017-0406-6.

10. Taşkınlar H, Bahadır GB, Erdoğan C, Yiğit D, Avlan D, Naycı A. A Diagnostic Dilemma for the Pediatrician: Radiolucent Tracheobronchial Foreign Body. Pediatr Neonatol. 2017;58(3):264-269. doi: 10.1016/j.pedneo.2016.07.003.

11. Lovrenski J, Vilotijević Dautović G, Lovrenski A. Reduced or Absent "Lung Sliding" - A Novel Lung Ultrasound sign of Pediatric Foreign Body Aspiration. J Ultrasound Med. 2019;38(11):3079-82. doi: 10.1002/jum.14988.

12. Chen X, Zhang C. Foreign body aspiration in children: Focus on the impact of delayed treatment. Int J Pedi- 
atr Otorhinolaryngol. 2017;96:111-5. doi: 10.1016/j. ijporl.2017.03.013.

13. Zhou J, Shang WY, Huang ZH, Liu YQ, Sun C, Shen XF, et al. Influential factors for visit time for tracheobronchial foreign bodies in pediatrics. Eur Arch of Otorhinolaryngol. 2020;277:505-9. doi: 10.1007/s00405-019-05700-5. Epub 2019 Oct 24.
14. Brkić F, Umihanić Š. Tracheobronchial foreign bodies in children Experience at ORL clinic Tuzla, 1954-2004. Int J Pediatr Otorhinolaryngol. 2007;71(6):909-15. Epub 2007 Apr 3.

15. Holan G, Ram D. Aspiration of an avulsed primary incisor. A case report. Int J Pediatr Dent. 2000;10(2):150-2. 